\title{
Les recommandations de la C.I.P.R. Application au cas particulier des mines d'uranium $(*)$
}

\author{
H. JAMMET $(* *)$ et M. DOUSSET
}

(Manuscrit reçu le 31 octobre 1980)

\begin{abstract}
RÉSUMÉ
Après avoir rappelé l'état actuel des connaissances sur les effets pathologiques des rayonnements, cette étude analyse les différents concepts élaborés par la Commission pour établir une expression quantitative correcte du détriment pour la santé. Elle s'attache ensuite à définir les trois impératifs fondamentaux de la doctrine de protection de l'homme : justification, optimisation et limites de dose individuelle. La troisième partie est consacrée au cas particulier des nuisances radiologiques telles qu'elles existent dans les mines d'uranium et à la façon dont les impératifs fondamentaux peuvent s'appliquer dans ce cas. Une place particulière est donnée aux limites fixées par le C.I.P.R. et à la réglementation française pour l'exposition aux produits de filiation du radon 222. La recommandation publiée dans le communiqué de mars 1980 de la C.I.P.R. sur l'exposition professionnelle au radon 222 et à ses produits de filiation est analysée.
\end{abstract}

\section{ABSTRACT}

After a review of the present state of knowledge on the pathological effects of ionizing radiation, an analysis is made of the different concepts developed by the Commission in order to establish a correct quantitative expression of the detriment to health. The three fundamentals of the philosophy of human protection are then defined: justification, optimization and individual dose limits. The third part is devoted to the case of radiological hazards met in uranium mines and the application of the basic principles in this particular case. The limits set up by I.C.R.P. and french regulations to the exposure to radon 222 daughter products are especially developed. The recommendation on radon 222 and its daughter products published in the I.C.R.P. statement issued in March 1980 is also analysed.

(*) Session d'étude sur la protection contre les rayonnements lors de l'exploitation et du traitement des minerais d'uranium, Vassivière, 22-24 septembre et 18-21 novembre 1980.

(**) Commissariat à l'Énergie atomique, Institut de Protection et de Sûreté nucléaire, Département de Protection, B. P. ${ }^{\circ} 6,92260$ Fontenay-aux-Roses. 


\section{INTRODUCTION}

Cette étude se propose de rappeler les grands principes de la protection de l'homme contre les rayonnements ionisants et de préciser comment ces principes s'appliquent, avec certaines difficultés particulières, au cas spécifique de la protection dans les mines d'uranium. Cet exposé comprendra trois grandes parties :

1. Les bases sur lesquelles s'appuie la doctrine de la Commission internationale de protection radiologique (C.I.P.R.).

2. Les impératifs fondamentaux de cette doctrine.

3. L'application aux risques spécifiques des mines d'uranium.

\section{BASES SUR LESQUELLES S'APPUIE LA DOCTRINE DE LA C.I.P.R.}

\subsection{LA C.I.P.R.}

La protection de l'homme contre les rayonnements ionisants est assurée dans chaque pays par l'application de dispositions réglementaires qui se fondent toutes sur une doctrine élaborée au niveau international par la C.I.P.R., commission créée en 1928 par le Congrès international de radiologie.

Depuis sa création, cette Commission s'est constamment tenu informée du développement des connaissances sur les effets pathologiques des rayonnements et des substances radioactives; elle a approfondi sa réflexion sur la démarche morale, plus ou moins consciente, de l'homme face aux grandes entreprises scientifiques, techniques ou industrielles qui impliquent toujours avec les avantages (connaissances, ressources, bien-être...) qu'elles apportent à l'humanité, des aspects négatifs comportant souvent des dommages difficiles à éviter pour la santé de l'homme.

Ce long effort d'approfondissement l'a conduite à élaborer une doctrine de la protection de l'homme contre les rayonnements ionisants dont l'expression la plus récente a fait l'objet de sa Publication 26 parue en 1977 [1]. Cette doctrine est fondée sur la connaissance que nous avons des effets pathologiques des rayonnements sur l'homme. Depuis la dernière guerre mondiale, un nombre considérable d'informations ont été acquises et, bien que certains domaines demandent encore à être éclaircis, les certitudes acquises sont suffisantes pour fonder une règle de conduite prudente.

\subsection{LES EFFETS PATHOLOGIQUES DES RAYONNEMENTS}

Rappelons brièvement que, du point de vue des lois qui régissent leur apparition, les effets pathologiques des rayonnements peuvent appartenir à deux grandes catégories :

- les effets non stochastiques (non aléatoires);

- les effets stochastiques (ou aléatoires). 
Les premiers apparaissent, souvent dans des délais assez brefs après l'exposition aux rayonnements, avec une gravité qui dépend de l'importance et des modalités de l'irradiation, toutes les fois que l'énergie communiquée par unité de masse de tissu (dose absorbée) dépasse un certain seuil.

Au-dessous de ce seuil ces effets n'apparaissent jamais. Les atteintes plus ou moins graves de la peau, le mal des rayons, la cataracte, les dommages vasculaires, les insuffisances respiratoires, la nécrose ou la fibrose des tissus sont des exemples de cette catégorie d'effets.

Les seconds sont toujours tardifs (plusieurs années ou dizaines d'années après l'exposition), leur gravité ne dépend pas de la dose absorbée et ils n'apparaissent, en général, que chez quelques-uns des individus exposés aux rayonnements, le nombre de cas observés diminuant avec la dose; de plus, comme toutes ces affections apparaissent chacune avec une certaine fréquence spontanée chez les individus non soumis à l'action des rayonnements, il est impossible, avec les méthodes statistiques qui sont les seuls moyens de connaissance actuellement à notre disposition, d'affirmer l'existence ou la nonexistence d'un seuil et de préciser la forme de la relation dose-fréquence d'apparition à faible dose. La leucémie, certains cancers (en particulier le cancer du poumon chez les mineurs de certaines mines d'uranium) et les effets génétiques appartiennent à cette catégorie.

Il découle directement des différences qui existent ainsi entre les lois d'apparition de ces deux catégories d'effets que, si pour les effets dits " non stochastiques " il est possible d'assurer une protection absolue de l'homme en fixant pour les doses reçues des limites à un niveau suffisamment bas, il n'en est pas de même pour les effets stochastiques pour lesquels la prudence a conduit la C.I.P.R. à admettre l'hypothèse que des doses faibles, même très faibles, entraînent une augmentation de la probabilité d'apparition de ces effets. Il y a donc là un certain risque hypothétique à accepter, mais en remarquant qu'il est toujours possible de réduire ce risque à un niveau aussi bas que l'on veut. Il suffit pour cela de réduire les doses car, dans le domaine des doses faibles, la relation dose-effet est toujours, quelle que soit sa forme exacte, une fonction qui décroît lorsque la dose décroît.

\subsection{LES CONCEPTS NÉCESSAIRES A L'EXPRESSION QUANTITATIVE DES RISQUES}

Pour exprimer de façon quantitative les relations qui existent entre les phénomènes physiques mesurables et le risque pour l'homme, compte tenu des différentes modalités d'exposition aux rayonnements, il est nécessaire de définir les différents concepts qui se sont progressivement dégagés de la réflexion sur les innombrables informations rassemblées dans ce domaine.

\subsubsection{Dose absorbée}

Définie comme une sorte de densité d'énergie communiquée aux tissus par les rayonnements, c'est une grandeur physique que l'on peut calculer, parfois mesurer, qui s'exprime, dans le système international d'unités, en grays (Gy). Un gray est égal à $1 \mathrm{~J} / \mathrm{kg}$. On emploie aussi, pour ne pas rompre brutalement avec le passé, une unité cent fois plus petite : le rad. 
Pour se rendre compte de ce que représentent ces unités, notons que la radioactivité naturelle entraîne en moyenne pour l'homme une dose absorbée de $0,1 \mathrm{rad}$ par an ou un millième $\left(10^{-3}\right)$ de gray par an, avec d'ailleurs des variations régionales ou locales importantes (de 0,05 à 0,4 rad et plus par an ).

\subsection{2. Équivalent de dose}

L'expérience montre que la dose absorbée est un paramètre insuffisant pour décrire et prévoir les effets des divers rayonnements ionisants. Aussi, des facteurs de pondération doivent-ils être introduits pour tenir compte de la nocivité propre à chaque type de rayonnement et, éventuellement, de toute sorte de modalités d'irradiation (étalement dans le temps, fractionnement de la dose, etc.). Le seul facteur de pondération actuellement défıni par la C.I.P.R. se rapporte à la nature et parfois à l'énergie des rayonnements dans le cas où les doses sont relativement faibles (de l'ordre de grandeur des limites de dose dont il sera parlé plus loin). Ce facteur se dénomme facteur de qualité $Q$ du rayonnement.

La dose absorbée pondérée par le facteur de qualité :

$$
H=D \cdot Q
$$

devient " l'équivalent de dose », il s'exprime en sieverts (Sv) ou en rems selon que la dose absorbée est exprimée en grays ou en rads.

\subsection{3. Équivalent de dose et effets non stochastiques}

L'une des premières indications qui se dégagent de nos connaissances sur les effets non stochastiques des rayonnements est que les différents seuils d'apparition des effets de cette catégorie sont beaucoup plus élevés (d'un facteur 3 à 5) pour une irradiation chronique (étalée dans le temps) que pour une irradiation aiguë.

La C.I.P.R. pense que, pour tous les tissus de l'organisme à l'exception des cristallins, un équivalent de dose annuel de $0,5 \mathrm{~Sv}$ (50 rems) répété durant toute la vie professionnelle (40 ans) entraînerait une exposition totale nettement inférieure au seuil d'apparition de ces effets.

\subsection{4. Équivalent de dose et effets stochastiques}

Pour les effets stochastiques, en l'absence de certitude sur l'existence ou la non existence d'un seuil ainsi que sur la forme exacte que peut avoir, à faible dose et faible débit de dose, la relation dose-effet, la C.I.P.R. a admis que pour chaque organe ou tissu irradié la probabilité de l'effet éventuel correspondant (ou plus exactement le produit de cette probabilité par un facteur exprimant la gravité de l'effet, produit appelé détriment) était proportionnelle à l'équivalent de dose reçu au niveau de cet organe ou tissu. Le coefficient de proportionnalité, est appelé facteur de risque. Ces facteurs ont été évalués avec prudence à partir des données obtenues par observation sur l'homme [2] et expérimentation sur l'animal dans la gamme des doses où l'action des rayonnements est suffisante pour que se dégagent des résultats statistiquement significatifs.

Les valeurs retenues par la C.I.P.R., exprimées en probabilité par sievert et par vie, sont présentées dans le tableau I. 
TABLEAU I

\begin{tabular}{|c|c|c|c|}
\hline Organes ou tissus & Effets pathologiques & $\begin{array}{c}\text { Facteur } \\
\text { de risque }\left({ }^{1}\right)\end{array}$ & $W_{T}\left({ }^{4}\right)$ \\
\hline 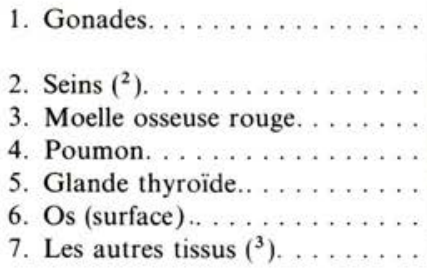 & $\begin{array}{l}\text { Effets génétiques pour les deux } \\
\text { premières générations } \\
\text { Mortalité par cancer } \\
\text { Mortalité par leucémie } \\
\text { Mortalité par cancer } \\
\text { Mortalité par cancer } \\
\text { Mortalité par cancer } \\
\text { Mortalité par cancer }\end{array}$ & $\begin{array}{l}0,40 \cdot 10^{-2} \\
0,25 \cdot 10^{-2} \\
0,20 \cdot 10^{-2} \\
0,20 \cdot 10^{-2} \\
0,05 \cdot 10^{-2} \\
0,05 \cdot 10^{-2} \\
0,50 \cdot 10^{-2}\end{array}$ & $\begin{array}{l}0,25 \\
0,15 \\
0,12 \\
0,12 \\
0,03 \\
0,03 \\
0,30\end{array}$ \\
\hline & & $1,65 \cdot 10^{-2}$ & 1,00 \\
\hline
\end{tabular}

(1) Le facteur de risque est exprimé par sievert et par vie.

$\left({ }^{2}\right)$ La valeur du facteur de risque correspondant est une valeur moyenne pour les deux sexes.

$\left({ }^{3}\right)$ "Les autres tissus " doivent s'entendre de la façon suivante :

a) les mains, les avant-bras, les pieds, les chevilles, la peau et les cristallins en sont exclus;

b) un facteur de risque de $0,1 \cdot 10^{-2}$ sera pris en considération pour chacun des 5 organes ou tissus restants pour lesquels les équivalents de dose sont les plus élevés. Le risque pour les autres tissus pourra être négligé.

( $\left.{ }^{4}\right) W_{T}=$ Détriment relatif au tissu $T /$ Détriment total.

\subsection{5. Équivalent de dose effectif}

Il n'est pas exclu que l'irradiation de plusieurs organes ou tissus ait un effet résultant quelque peu différent de la somme des effets obtenus après une irradiation partielle de chacun d'eux. Cependant, comme les facteurs de risque retenus par la C.I.P.R. résultent d'études portant à la fois sur des irradiations partielles et des irradiations totales de l'organisme humain, ils tiennent compte de l'ensemble des informations acquises sur l'importance relative des différents tissus dans le phénomène général d'induction des affections malignes par les rayonnements ionisants [2]. Dès lors, on peut penser qu'avec des facteurs de risque ainsi évalués, il est licite d'admettre la règle d'additivité des risques et de calculer, dans le cas d'une irradiation uniforme de tous les tissus de l'organisme, le facteur de risque total comme la somme des facteurs de risque retenus pour chacun des tissus ou groupes de tissus énumérés dans le tableau I. Ceci permet de préciser (dernière colonne du tableau I) la fraction $W_{T}$ du risque total qui est due à l'irradiation du tissu $T$.

On peut donc considérer que le paramètre fondamental dont la valeur numérique est directement proportionnelle au risque total éventuel est la somme :

$$
W_{1} H_{1}+W_{2} H_{2}+\ldots+W_{7} H_{7}=\sum_{T} W_{T} H_{T}=H_{E}
$$

appelée équivalent de dose effectif et dans laquelle $H_{1}, H_{2}, \ldots, H_{7}$ représentent les équivalents de dose reçus respectivement par les organes ou tissus $1,2, \ldots, 7$ du tableau I. 
Aussi bien dans le cas d'une exposition uniforme de l'organisme que dans celui où les différents tissus reçoivent des doses diverses, l'équivalent de dose effectif est le paramètre général qui donne une mesure du risque total éventuel pour l'individu exposé.

Le fait qu'ait été ainsi établie une relation entre l'enveloppe du risque total éventuel et un paramètre dont on peut assurer la maîtrise en réduisant à volonté directement (irradiation externe) ou indirectement (contamination interne) les doses au niveau des différents tissus, permet de réduire le détriment éventuel lié aux effets stochastiques à un niveau aussi faible qu'on veut.

\subsection{6. Équivalent de dose engagé}

La façon dont le concept d'équivalent de dose effectif s'applique dans le cas de la contamination interne de l'organisme présente une particulière importance car l'apport de substance radioactive par la voie respiratoire constitue l'une des principales modalités d'atteinte des travailleurs dans l'exploitation des installations du cycle du combustible.

Lorsqu'un radionucléide a pénétré dans l'organisme (par inhalation, par ingestion ou par blessure), la fraction de la quantité ainsi incorporée qui parvient aux différents organes ou tissus est extrêmement variable selon la nature chimique du radionucléide, la forme physico-chimique sous laquelle il se trouve et selon la voie d'entrée dans l'organisme. Si nous considérons un organe dans lequel une certaine quantité du radionucléide est parvenue, le séjour de ce radionucléide dans cet organe sera une fonction décroissante plus ou moins complexe du temps, dépendant de l'élimination biologique et de la décroissance radioactive.

Le débit d'équivalent de dose (équivalent de dose par unité de temps) au niveau de cet organe variera, en première approximation, comme varie l'activité du radionucléide présent dans cet organe, et c'est l'équivalent de dose total délivré à cet organe depuis l'incorporation du radionucléide jusqu'à la fin de la vie (en pratique, on le calcule sur les 50 années qui suivent l'incorporation) qui revêt une signification; on l'appelle équivalent de dose engagé et on le désigne par $H_{50 T}$. La C.I.P.R. admet implicitement que le risque qui lui est associé est pratiquement indépendant (au moins au niveau des faibles doses) de la façon dont cet équivalent de dose total est délivré dans le temps.

Il est bon de noter pour éviter toute confusion que, dans l'expression souvent employée équivalent de dose engagé en un temps donné $t$ (1 an par exemple), ce temps $t$ est la durée pendant laquelle s'effectuent les incorporations (inhalation ou ingestion) et non le temps sur lequel le débit d'équivalent de dose à l'organe est intégré qui, lui, est toujours égal à 50 ans.

On démontre d'ailleurs que, pour les quantités pondérales toujours très faibles mises en jeu dans les cas courants de contamination radioactive, l'incorporation d'une même activité $A$ entraîne toujours le même équivalent de dose engagé quel que soit le temps $t$ pendant lequel se produit l'incorporation de $A$ et quelle que soit également la modalité aiguë, continue ou fractionnée de l'incorporation. 
Pour appliquer la notion d'équivalent de dose effectif à la contamination interne, il suffira d'introduire les équivalents de dose engagés relatifs à chaque organe dans l'expression :

$$
\sum W_{T} H_{T},
$$

qui définit l'équivalent de dose effectif. On obtient ainsi l'équivalent de dose effectif engagé :

$$
H_{E S 0} \quad \text { qui est égal à } \sum W_{T} H_{50 T} \text {. }
$$

La connaissance que nous avons du métabolisme des différents éléments permet de calculer pour chaque radionucléide les équivalents de dose effectifs engagés qui correspondent à l'incorporation par voie digestive ou par voie respiratoire d'une quantité donnée de radionucléide.

A titre d'exemple le tableau II donne, pour l'inhalation de $1 \mathrm{~Bq}$ des radionucléides qui se trouvent dans les poussières de minerai d'uranium, l'équivalent de dose effectif engagé ainsi que l'équivalent de dose engagé au niveau de l'organe (ou tissu) le plus irradié.

\section{TABLEAU II}

ÉQUIVALENTS DE DOSE ENGAGÉS

\begin{tabular}{|c|c|c|c|c|}
\hline Radionucléides & $\begin{array}{c}\text { Rayonnement } \\
\text { émis }\end{array}$ & $\begin{array}{c}\text { Classe } \\
\text { de } \\
\text { solubilité }\left({ }^{2}\right)\end{array}$ & $\begin{array}{c}H_{E, 50} \\
\left(\mathrm{~Sv} \cdot \mathrm{Bq}^{-1}\right)\end{array}$ & $\begin{array}{c}H_{T, s o} \\
\left(\mathrm{~Sv} \cdot \mathrm{Bq}^{-1}\right)\end{array}$ \\
\hline${ }^{238} \mathrm{U} \ldots \ldots \ldots$ & $\alpha$ & Y & $3,2 \cdot 10^{-5}$ & $2,7 \cdot 10^{-4}$ poumon \\
\hline${ }^{234} \mathrm{Th}+{ }^{234} \mathrm{~Pa} . \ldots \ldots \ldots$ & $\beta$ & $\mathrm{Y}$ & $8,9 \cdot 10^{-9}$ & $6,4 \cdot 10^{-8}$ poumon \\
\hline${ }^{234} \mathrm{U} \ldots \ldots \ldots \ldots \ldots$ & $\alpha$ & Y & $3,6 \cdot 10^{-5}$ & $3 \cdot 10^{-4}$ poumon \\
\hline${ }^{230}$ Th. . . . . . . . . & $\alpha$ & $\mathrm{Y}$ & $7.10^{-5}$ & $8,7 \cdot 10^{-4}$ os $\left({ }^{3}\right)$ \\
\hline${ }^{226} \mathrm{Ra} . \ldots \ldots \ldots \ldots$ & $\alpha$ & W & $2,1 \cdot 10^{-6}$ & $1,6 \cdot 10^{-5}$ poumon \\
\hline${ }^{210} \mathrm{~Pb} . \ldots \ldots \ldots \ldots$ & $\beta$ & D & $4,8 \cdot 10^{-6}\left({ }^{4}\right)$ & $7 \quad .10^{-5}$ os $\left({ }^{3}\right)\left({ }^{4}\right)$ \\
\hline${ }^{210} \mathrm{Bi} \ldots \ldots \ldots \ldots \ldots$ & $\beta$ & W & $5,3 \cdot 10^{-8}\left({ }^{4}\right)$ & $4,3 \cdot 10^{-7}$ poumon \\
\hline${ }^{210}$ Po. . . . . . . . & $\alpha$ & W & $2,1 \cdot 10^{-6}$ & $1,3 \cdot 10^{-5}$ poumon \\
\hline
\end{tabular}

POUR L'INCORPORATION DE $1 \mathrm{~Bq}\left({ }^{1}\right)$ PAR VOIE RESPIRATOIRE

(1) L'unité d'activité dans le système international d'unité est le becquerel ( $\mathrm{Bq})$ égal à 1 désintégration par seconde. Le curie $(\mathrm{Ci})$ vaut $3,7 \cdot 10^{10} \mathrm{~Bq}$.

$\left({ }^{2}\right)$ Les différentes formes chimiques sous lesquelles se présentent les radionucléides sont réparties en 3 classes selon leur plus ou moins grande aptitude à franchir la barrière pulmonaire (solubilité ou diffusibilité). Dans l'ordre de la plus grande diffusibilité à la plus faible on note ces classes D, W, Y.

$\left({ }^{3}\right)$ Os $=$ Cellules tapissant la surface des os.

( $\left.{ }^{4}\right)$ Pour ${ }^{210} \mathrm{~Pb}$ et ${ }^{210} \mathrm{Bi}$ les valeurs sont tirées de NRPB-R-82 [5]. Pour les autres radionucléides, elles proviennent de la Publication I.C.R.P. 30 (partie 1 et supplément à la partie 1) [4].

vOL. $16-\mathrm{N}^{\circ} 1$ 


\subsection{7. Équivalent de dose collectif}

Enfin, il est un autre concept dont il faut préciser la signification c'est celui d'équivalent de dose collectif qui joue un rôle particulièrement important dans le processus d'optimisation dont on parlera plus loin.

Il arrive, en effet, que l'on ait à considérer le risque collectif (pour un groupe donné de travailleurs ou de personnes du public) qui résulte d'une activité industrielle, scientifique, etc. pouvant entraîner une exposition de l'homme aux rayonnements. Ce risque collectif est constitué, au niveau des faibles doses, par l'ensemble des effets stochastiques éventuels qui peuvent survenir dans le groupe de travailleurs ou de populations que l'on considère. Dans l'hypothèse prudente admise par la C.I.P.R. d'une relation linéaire et sans seuil, à faible dose, entre l'effet et l'équivalent de dose effectif $\left(H_{E}\right)$, ce risque collectif sera proportionnel à la somme des équivalents de dose effectifs reçus par chacune des personnes constituant ce groupe.

Pour un sous-groupe de $P$ personnes recevant la même dose $\left({ }^{1}\right) H_{E}$, l'équivalent de dose collectif sera égal au produit :

$$
P H_{E}
$$

et, si le groupe est constitué de plusieurs sous-groupes $1,2, \ldots, i$ dans chacun desquels la dose est $H_{E 1} H_{E 2} \ldots H_{E i}$ et le nombre de personnes $P_{1} P_{2} \ldots P_{i}$, l'équivalent de dose collectif sera défini par la somme :

$$
\sum_{i} P_{i} H_{E i}
$$

Cette nouvelle grandeur, appelée équivalent de dose collectif, somme de produits d'un nombre de personnes par un équivalent de dose effectif, s'exprime en hommes-sieverts ou hommes-rems.

\section{LES IMPÉRATIFS FONDAMENTAUX DE LA DOCTRINE DE LA C.I.P.R.}

C'est en se fondant sur l'ensemble des connaissances qui ont été rappelées plus haut concernant les effets pathologiques des rayonnements et en utilisant les différentes notions qui viennent d'être définies, que la C.I.P.R. a élaboré sa doctrine de la protection de l'homme contre les rayonnements.

Cette doctrine qu'elle a appelée le système de limitation des doses se ramène essentiellement à trois grands principes qui peuvent être désignés par les expressions :

- justification de l'action;

- optimisation de la protection;

- limite des doses individuelles.

(1) Pour alléger le texte le mot "dose » est souvent employé ici pour "équivalent de dose effectif $m$. 


\subsection{JUSTIFICATION DE L'ACTION}

Toute entreprise humaine qui peut entraîner une exposition de l'homme aux rayonnements doit être justifiée. La prise de décision doit être fondée sur une analyse coût-avantages montrant que le coût total de l'opération, y compris les dommages éventuels pour la santé de l'homme, évalués en tenant compte des moyens de protection optimisés mis en œuvre, est largement compensé par les avantages que la société et les individus retireront de cette activité.

Il est théoriquement possible de formaliser cette procédure d'analyse, mais les difficultés rencontrées pour exprimer quantitativement certains éléments du bilan ne permettent guère, à l'heure actuelle, d'utiliser le formalisme mathématique de sorte que l'analyse justificative comporte la plupart du temps beaucoup d'aspects qualitatifs, ce qui n'altère d'ailleurs en rien son efficacité.

Ce principe est tout à fait général et s'applique à toutes les activités humaines qu'elles soient à fin médicale, scientifique, industrielle, etc., qu'elles impliquent ou non l'utilisation des rayonnements ou des substances radioactives.

Dès lors, il est évident que lorsque pour atteindre une même fin plusieurs stratégies ou plusieurs moyens techniques sont en compétition et présentent des bilans " coût-avantages " différents, il faut choisir la solution pour laquelle le bilan est le meilleur.

Dans certaines activités, médicales ou scientifiques par exemple, la justification relève souvent de la responsabilité individuelle de celui qui décide et agit : c'est le cas du médecin ou du directeur de recherche qui décide d'employer une technique mettant en jeu des rayonnements ou des substances radioactives plutôt qu'une autre technique non radiologique mais jugée moins efficace ou moins adaptée.

Par contre, les grandes options, comme celles qui conduisent à promouvoir l'énergie nucléaire, donc à exploiter des mines d'uranium et à développer toutes les installations du cycle du combustible, ou celles qui aboutissent à l'autorisation générale de commercialiser certains appareils ou certaines techniques, sont du domaine des pouvoirs publics, et c'est à leur niveau que les études de justification sont faites et les décisions prises.

\subsection{Optimisation DE LA PROTECTION}

Le second principe a trait au niveau de protection de l'homme qu'il convient d'atteindre dans une entreprise dont la réalisation est justifiée par le bilan préalable dont on vient de parler, bilan qui, d'ailleurs, suppose acquis ce niveau optimal de protection.

Il est certain que dans le domaine de la protection des travailleurs et du public, on peut toujours faire mieux et qu'il suffit d'y mettre le prix pour améliorer sûreté et protection; le problème qui se pose ici est de déterminer le niveau auquel il faut s'arrêter. 
La Commission a énoncé le principe théorique suivant : les expositions doivent être maintenues aux niveaux les plus faibles auxquels on peut raisonnablement parvenir compte tenu des aspects économiques et sociaux. Pour déterminer ces niveaux, elle indique qu'on peut utiliser une analyse différentielle où l'on compare le gain sur le risque éventuel que court la santé de l'homme (effets stochastiques) avec le coût de l'amélioration de la protection qui permet d'obtenir ce gain. Comme ce coût augmente avec le niveau de protection que l'on veut améliorer, il arrive un moment où le gain sur le risque ne vaut pas le prix de l'amélioration de la protection envisagée : ce niveau de protection est le niveau optimal que l'on doit rechercher.

Ce principe a une portée générale; il doit s'appliquer aussi bien à l'exposition des malades dans l'utilisation à des fins médicales des rayonnements (le malade est alors à la fois le bénéficiaire de l'amélioration de la santé que doit lui apporter l'irradiation et le sujet qui en subira les éventuels dommages) qu'aux espositions professionnelles et aux expositions du public dans toutes les activités radiologiques et nucléaires.

Dans ce dernier cas, pour déterminer cet optimum, on tient principalement compte du risque sous sa forme collective pour lequel on a fait l'hypothèse prudente qu'il est proportionnel à l'équivalent de dose collectif défini précédemment. En général, le processus conduit donc, dans une situation concrète donnée, à un niveau de protection correspondant à un nombre optimal "d'hommes-sieverts " ou "d'hommes-rems ». On verra, plus loin, que cette façon de s'exprimer est difficilement applicable lorsqu'on a affaire aux produits de filiation du radon.

Dans le cas des mines d'uranium et des usines de traitement du minerai, les problèmes posés par l'impératif d'optimisation sont relativement complexes. Comme cela a été dit dans l'exposé précédent, on y trouve trois principaux risques radiologiques :

- l'irradiation externe par les rayonnements $\gamma$ émis par le minerai;

- la contamination par inhalation des produits de filiation du radon;

- la contamination par inhalation des poussières de minerai qui contiennent de nombreux radionucléides à vie plus ou moins longue.

C'est sur ces trois modalités d'atteinte de l'organisme que doivent porter d'une part les efforts tendant à diminuer les risques pour la santé et d'autre part la confrontation du résultat obtenu avec les inconvénients que présentent les moyens mis en œuvre.

Il est important de noter que ces inconvénients ne sont pas toujours des inconvénients financiers (inconvénients d'attribution de crédits, par exemple, dont on peut toujours discuter de savoir s'ils ne seraient pas mieux employés pour lutter contre telle nuisance plutôt que contre telle autre) mais parfois aussi des inconvénients pour la santé. C'est ainsi que l'expérience a montré que le principal moyen employé pour diminuer la concentration des produits de filiation du radon 222 dans l'air des mines souterraines d'uranium, la ventilation, ne pouvait pas être augmentée indéfiniment sous peine d'entraîner des affections pulmonaires chez les mineurs. 
Il faut donc trouver un débit optimal qui se présente comme un compromis entre deux exigences contradictoires. C'est là un bon exemple d'application de l'impératif d'optimisation.

Il est certain, cependant, qu'en règle générale, les contraintes techniques et les coûts financiers jouent un rôle important dans l'analyse différentielle qui permet de déterminer le niveau optimal de protection et qu'il n'est pas possible, en particulier, de faire abstraction, comme le voudrait une certaine vision des choses, du rôle que jouent les prix de revient dans la réalité de la concurrence internationale.

La butée absolue, en matière de protection, celle dont le franchissement ne saurait être justifié ni par des raisons économiques, ni par des raisons sociales, est le respect des limites de dose individuelle dont il va être traité maintenant. Cependant, dans la hiérarchie des grands principes de protection, la C.I.P.R. ne situe les limites qu'au troisième rang pour bien marquer la prééminence de l'optimisation qui doit conduire à un niveau de protection, variable certes d'une branche à l'autre du nucléaire, mais toujours bien meilleur que le simple respect des limites.

\subsection{LiMites DE DOSE INDIVIDUELLE}

D'une façon générale, le niveau optimal de protection assure pour un groupe donné de personnes une limitation du dommage collectif, par exemple une limitation du nombre total de cas de leucémies, cancers et effets génétiques qui pourraient survenir dans l'avenir (si les faibles doses ont un effet), mais il ne tient pas compte, du moins théoriquement, de la distribution individuelle des doses et pourrait donc, à la limite, ne pas assurer une protection suffisante de l'individu contre ces risques éventuels. D'autre part, il faut remarquer qu'en particulier pour l'exposition du public, les personnes qui subissent les irradiations ne sont pas toujours celles qui bénéficient pleinement des avantages que procurent les activités industrielles, scientifiques ou autres qui sont la cause de leur exposition.

Il est donc nécessaire, pour l'exposition professionnelle et l'exposition du public, de limiter le risque individuel en fixant des limites aux équivalents de dose reçus par chaque personne.

Le respect de ces limites de dose individuelle, après que l'optimum de protection collective ait été assuré, est une garantie pour l'individu. Il constitue le troisième impératif de la doctrine de la C.I.P.R.

\subsubsection{Limites fondamentales primaires}

Se fondant sur plus de 30 ans de pratique dans les différentes activités où les rayonnements et les substances radioactives sont utilisés, la Commission a tenu à conserver les principales valeurs des limites déjà recommandées dans le passé, à savoir, pour une exposition uniforme de l'organisme entier :

- une limite de $0,05 \mathrm{~Sv}$ ou 5 rems pour les équivalents de dose reçus ou engagés en 1 an, lorsqu'il s'agit de l'exposition professionnelle;

- une limite dix fois plus faible dans le cas de l'exposition du public. 
Il est rare qu'on ait affaire à une irradiation vraiment uniforme de l'organisme; dans le cas de l'irradiation externe par des rayonnements pénétrants, on admet cette uniformité comme hypothèse simplificatrice et c'est la valeur maximale de la dose dans l'organisme qui est considérée comme reçue par tous les tissus et organes et qui, par conséquent, doit respecter la limite de dose individuelle de 0,05 ou 0,005 Sv par an.

$\mathrm{Au}$ niveau de la peau où les rayonnements peu pénétrants comme les rayonnements $\beta$ peuvent délivrer une dose qui s'ajoute à celle des rayonnements pénétrants $(\gamma$, neutrons) la limite est fixée à $0,5 \mathrm{~Sv}$ pour l'exposition professionnelle et à $0,05 \mathrm{~Sv}$ pour le public.

Dans le cas de la contamination interne, un certain nombre d'éléments ont une répartition à peu près uniforme (tritium, carbone, césium, ... par exemple) mais, dans la majorité des cas, la répartition entre les différents tissus ou organes est très inégale et les équivalents de dose engagés au niveau de ces divers tissus sont très différents les uns des autres.

La C.I.P.R. a recommandé alors, comme dans le cas plus général des irradiations partielles, deux limites qui doivent être respectées simultanément :

- la première, destinée à éliminer toute possibilité d'apparition d'effets non stochastiques, fixe une valeur maximale à l'équivalent de dose engagé par an au niveau des différents tissus ou organes. Sa valeur est $0,5 \mathrm{~Sv}$ (soit 50 rems) pour l'exposition professionnelle $(0,15 \mathrm{~Sv}$, soit 15 rems pour les cristallins $)$ et $0,05 \mathrm{~Sv}$ (soit 5 rems) pour l'exposition des personnes du public;

- la seconde garantit, pour les effets stochastiques, un niveau de risque éventuel acceptable pour l'individu, en fixant une valeur maximale pour l'équivalent de dose effectif engagé par an. Sa valeur est $0,05 \mathrm{~Sv}$ ( 5 rems) pour l'exposition professionnelle et $0,005 \mathrm{~Sv}(0,5 \mathrm{rem})$ pour l'exposition des personnes du public.

Ces limites s'appliquent aux doses annuelles totales résultant des diverses modalités d'exposition de l'individu aux rayonnements (irradiation externe et contamination interne par un ou plusieurs radionucléides). Dans la pratique, il n'est pas toujours facile de procéder à l'évaluation de la dose totale et on a recours à une formule de cumul des risques dont l'application au cas des mines d'uranium sera précisée dans la troisième partie de ce texte.

Comme il a été dit plus haut, ces valeurs ont été retenues, on pourrait dire reconduites, en 1977, par la C.I.P.R., parce que leur application, sous une forme quelque peu différente et moins précise, a donné toute satisfaction à l'égard de la protection de l'homme au cours des 30 dernières années qui ont vu un développement considérable des activités médicales, scientifiques et industrielles dans le domaine radiologique et nucléaire.

Cependant, se fondant sur les connaissances actuellement acquises sur les effets pathologiques des rayonnements, la C.I.P.R. a tenu à faire une estimation chiffrée du risque que pourrait entraîner l'application du système de limitation des doses qu'elle recommande avec les limites précédemment indiquées dans l'hypothèse prudente où les relations dose-effet seraient, à faible dose, linéaires et sans seuil avec les pentes, ou facteurs de risque indiqués au tableau I. 
Pour l'exposition professionnelle, l'application de ces limites de dose individuelle associée à la procédure d'optimisation conduit à un risque moyen (hypothétique) qui est, au plus, de l'ordre de grandeur du risque (réel) dû aux accidents du travail et aux maladies professionnelles dans les professions reconnues statistiquement comme présentant un bon degré de sûreté (risque de l'ordre de 1 cas mortel pour 10000 et par an).

Il s'agit d'un risque moyen calculé sur un ensemble professionnel comparable à ceux qui ont été choisis comme référence et pour lesquels le risque moyen est connu à partir des données statistiques établies dans les différents pays. De tels ensembles sont constitués, par exemple, par les travailleurs de l'industrie métallurgique, chimique, ceux de l'industrie du livre, des textiles, etc. Ces ensembles industriels comprennent, en général, de multiples branches correspondant aux différentes étapes de l'élaboration des produits finis comme, par exemple pour la métallurgie : le traitement des minerais métalliques, la métallurgie des alliages, la métallurgie de transformation, etc. qui peuvent présenter des risques nettement différents de la valeur moyenne que les statistiques ont établie pour l'ensemble professionnel. Il est bien évident qu'il ne peut pas en être autrement pour les différentes branches industrielles qui correspondent aux différentes étapes du cycle du combustible nucléaire.

Pour l'exposition du public, le système de limitation des doses assure un niveau de risque éventuel nettement inférieur à celui que nous acceptons d'encourir lorsque nous nous livrons aux différentes activités de la vie quotidienne pour lesquelles la sécurité des personnes n'est pas laissée à la libre initiative de celles-ci (les risques alors acceptés peuvent être considérables) mais est assurée par une réglementation établie par les pouvoirs publics comme, par exemple, la sécurité des voyageurs empruntant les moyens de transport en commun.

\subsubsection{Limites fondamentales secondaires}

Les limites fondamentales dont il vient d'être question ont un caractère quelque peu abstrait quand il s'agit de les appliquer au cas de la contamination interne de l'organisme, car les équivalents de dose au niveau des différents tissus ne sont alors ni directement mesurables, ni directement évaluables comme dans le cas de l'irradiation externe où l'on admet que la valeur maximale de la dose s'applique à tous les tissus.

Compte tenu des caractéristiques physiques, chimiques, radioactives, etc. que présente le radionucléide inhalé ou ingéré, on relie cependant, comme cela a été indiqué en 1.3.6, l'activité incorporée et les équivalents de dose engagés qui résulteront de cette incorporation au niveau de chaque organe. On peut alors déduire de chacune des valeurs limites qui ont été respectivement fixées pour l'équivalent de dose engagé au niveau de l'organe le plus irradié d'une part et pour l'équivalent de dose effectif engagé d'autre part, les valeurs limites correspondantes pour l'activité incorporée. Il va de soi que la plus sévère de ces deux valeurs devra être retenue et constituera la limite annuelle d'incorporation. 
Il s'agit là d'une limite qui est également une limite fondamentale, mais qui présente un caractère secondaire par rapport aux limites d'équivalent de dose, car elle se déduit de celles-ci par l'intermédiaire de modèles métaboliques nécessairement quelque peu formalisés.

Le tableau III indique, pour les radionucléides mentionnés dans le tableau II, les limites annuelles d'incorporation par inhalation pour l'exposition professionnelle.

TABLEAU III

LIMITES ANNUELLES D'INCORPORATION (LAI) PAR INHALATION ET LIMITES DÉRIVÉES DE CONCENTRATION DANS L'AIR (LDCA) POUR L'EXPOSITION PROFESSIONNELLE

\begin{tabular}{|c|c|c|c|}
\hline Radionucléides & $\begin{array}{l}\text { LAI } \\
(\mathrm{Bq})\end{array}$ & $\begin{array}{c}\text { LDCA } \\
\left(\mathrm{Bq} \cdot \mathrm{m}^{-3}\right)\end{array}$ & $\begin{array}{c}\text { Critère } \\
\text { prépondérant }\end{array}$ \\
\hline${ }^{238} \mathrm{U}(\alpha)$. & $2.10^{3}\left({ }^{4}\right)$ & $7.10^{-1}$ & $H_{E}=0,05 \mathrm{~Sv}\left({ }^{2}\right)$ \\
\hline${ }^{234} \mathrm{Th}+{ }^{234} \mathrm{~Pa} \ldots$ & $6.10^{6}$ & $2.10^{3}$ & $H_{E}=0,05 \mathrm{~Sv}$ \\
\hline${ }^{234} U(\alpha) \ldots \ldots$ & $1.10^{3}\left({ }^{4}\right)$ & $6.10^{-:}$ & $H_{E}=0,05 \mathrm{~Sv}$ \\
\hline${ }^{230} \operatorname{Th}(\alpha) . \ldots$. & $6.10^{2}$ & $2.10^{-1}$ & $H_{\mathrm{os}}=0,5 \mathrm{~Sv}\left({ }^{3}\right)$ \\
\hline${ }^{226} \mathrm{Ra}(\alpha)$. & $2.10^{4}$ & $1.10^{1}$ & $H_{E}=0,05 \mathrm{~Sv}$ \\
\hline${ }^{210} \mathrm{~Pb} \ldots$. & $7.10^{3}\left({ }^{1}\right)$ & $3.10^{\circ}\left({ }^{1}\right)$ & $H_{\mathrm{os}}=0,5 \mathrm{~Sv}$ \\
\hline${ }^{210} \mathrm{Bi}$ & $1.10^{6}\left({ }^{1}\right)$ & $4.10^{2}\left({ }^{1}\right)$ & $H_{E}=0,05 \mathrm{~Sv}$ \\
\hline${ }^{210} \mathrm{Po}(\alpha)$. & $2.10^{4}$ & $1.10^{1}$ & $H_{E}=0,05 \mathrm{~Sv}$ \\
\hline
\end{tabular}

${ }^{1}$ ) Valeurs provisoires extraites de NRPB-R-82 en attendant la parution des parties 2 et 3 de la Publication 30 de la C.I.P.R.

( $\left.{ }^{2}\right) H_{E}=$ Équivalent de dose effectif $(\$ 1.3 .5)$.

$\left({ }^{3}\right) H_{\text {os }}=$ Équivalent de dose au niveau des cellules tapissant la surface des os.

${ }^{(4)}$ Avant arrondissement, ces valeurs sont 1590 et 1328 , ce qui explique les valeurs proches des LDCA.

\subsubsection{Limites dérivées}

A partir de la limite annuelle d'incorporation, on calcule, en tenant compte des caractéristiques respiratoires moyennes de l'homme, l'activité volumique de l'air qui, inhalé durant toutes les heures de travail de l'année (2 000 heures avec un débit respiratoire de $1,2 \mathrm{~m}^{3}$ d'air par heure) correspondrait à la limite annuelle d'incorporation par voie pulmonaire. Ces activités volumiques sont dites limites dérivées de concentration dans l'air. Elles sont également indiquées au tableau III pour les radionucléides de la chaîne de l'uranium 238, à l'exclusion du radon 222 et de ses produits de filiation à vie courte. 


\section{APPLICATION DES RECOMMANDATIONS DE LA C.I.P.R. AUX NUISANCES DES MINES D'URANIUM}

\section{1. Les NUISANCES RADIOlOgiQues}

Comme cela a déjà été rappelé à titre d'exemple, les trois principales nuisances radiologiques liées à l'extraction et au traitement des minerais d'uranium sont :

- Une irradiation externe par les rayonnements émis par les différents radionucléides de la chaîne de l'uranium présents dans les roches en place ou abattues et dans l'air. Ces rayonnements entraînent une certaine dose au niveau de la peau $(\beta+\gamma)$ et une certaine dose en profondeur $(\gamma)$. On admet que cette dernière est la même pour tous les tissus ou organes internes du corps et que sa valeur est celle qui est mesurée par le dosimètre individuel porté par le travailleur, ce dosimètre ayant été étalonné pour donner la valeur maximale de la dose en profondeur. La dose à la peau est un peu supérieure $(+20 \%$ environ $)$ à cette dose en profondeur.

- Une irradiation interne des tissus pulmonaires principalement, due à l'inhalation des radionucléides à vie longue présents dans les poussières de minerai en suspension dans l'air. On peut admettre, en première approximation, que ces divers radionucléides sont en équilibre séculaire avec l'uranium 238.

- Une irradiation interne, des tissus pulmonaires principalement, due à l'inhalation des produits de filiation à vie courte du radon 222 . Ce gaz, émetteur $\alpha$ engendré par le radium 226, se dégage des roches uranifères et des eaux d'exhaure. Sa période propre est assez longue $(3,8 \mathrm{j})$ mais ses enfants : polonium 218 (3 $\mathrm{min})$, plomb 214 (27 $\mathrm{min})$, bismuth 214 (20 $\mathrm{min})$, polonium $214(164 \mu \mathrm{s})$ se mettent rapidement en équilibre avec lui dans les atmosphères peu ventilées et ce sont eux qui dans l'appareil respiratoire entraînent les doses les plus importantes, la contribution du radon étant négligeable.

Dans les mines souterraines, la nuisance " produits de filiation du radon " est le plus souvent nettement prédominante, malgré un puissant aérage; l'irradiation externe, dont l'importance varie avec la teneur du minerai, se situe en général entre 10 et $20 \mathrm{p}$. cent de la première; quant à l'inhalation des poussières, elle constitue une nuisance relativement faible.

Dans les mines à ciel ouvert, l'importance du radon et de ses produits de filiation est nettement atténuée et l'irradiation externe et les poussières prennent une importance relativement plus grande.

Dans les usines de traitement du minerai, le radon devient un risque mineur, sauf peut-être à certains postes de travail comme les postes de surveillance des silos de stockage avant concassage du minerai. Mais les risques d'irradiation externe et d'inhalation de poussières de minerai subsistent en de nombreuses zones et, en fin de procédé, s'ajoute le risque d'inhalation de composé pulvérulent d'uranium (uranate d'ammonium ou « Yellow cake »). 


\subsection{LA JUSTIFICATION}

Dans l'esprit de la C.I.P.R. le détriment pour la santé découlant des nuisances radiologiques qui viennent d'être rappelées, et auxquelles s'ajoutent les risques classiques des exploitations minières, ne peut être justifié que si le bilan total est nettement positif. Cependant, comme cela a été précisé précédemment, le bilan total ne peut être fait que dans la perspective d'ensemble du cycle de l'uranium et du développement du nucléaire avec tous les avantages attendus : production d'énergie, mise au point et application de techniques de pointe dans les différents domaines : médicaux, scientifiques, industriels, etc.

La justification est donc inséparable d'un choix politique dont la responsabilité revient aux pouvoirs publics dans chaque pays.

\subsection{L'OPTIMISATION DE LA PROTECTION}

Elle porte sur la mise en œuvre des principaux moyens de lutte contre la pollution atmosphérique : la réduction des sources de radon, l'aérage dans les mines souterraines, l'abattage des poussières et le développement des moyens mécaniques d'exploitation permettant de réduire l'effectif des travailleurs au fond.

Le développement des moyens collectifs de protection dans les mines d'uranium a été considérable depuis ces 20 dernières années dans tous les pays où l'on exploite des mines d'uranium et il paraît difficile d'aller beaucoup plus loin dans l'amélioration de l'efficacité des moyens classiques permettant de réduire la concentration en produits de filiation du radon et en poussières de minerais.

Certes, comme l'irradiation externe, l'irradiation interne par le radon et les poussières peut être réduite, si l'on se place au point de vue des doses individuelles, par une augmentation du nombre de travailleurs et une diminution du temps de travail au fond. Il en résulte, certes, une diminution des doses individuelles, mais la dose collective reste au minimum la même pour l'extraction d'une même quantité d'uranium et le prix de revient croît. La recherche d'un équilibre constitue l'un des problèmes les plus difficiles que pose l'exploitation des mines souterraines d'uranium. Cependant, il semble qu'une solution soit un effort vers une mécanisation plus poussée des opérations d'exploitation. Dans la mesure où la mise au point des techniques le permettrait, cette mécanisation entraînerait une diminution des effectifs exposés et conduirait à une diminution et de la dose collective et des doses individuelles. Encore faudrait-il que le coût de tels investissements soit compatible avec l'équilibre économique général que conditionnent de nombreux autres paramètres.

\subsection{LES LIMITES INDIVIDUELLES}

Les limites individuelles à respecter découlent de ce qui a été dit dans les paragraphes 2.3.1 à 2.3.3, sur les limites de dose fondamentales et sur les limites dérivées; cependant, la présence des trois modalités d'atteinte 
(irradiation $\gamma$, poussières, radon) entraîne que l'on tienne compte de ce qui a été appelé le cumul des risques et, d'autre part, les problèmes posés par la détermination de la limite d'incorporation pour les produits de filiation du radon appellent un assez long développement.

\subsubsection{L'irradiation externe}

Les équivalents de dose reçus au niveau de la peau étant de 20 p. cent environ supérieurs aux équivalents de dose en profondeur, l'irradiation superficielle ne pose aucun problème lorsque la limite de $0,05 \mathrm{~Sv} . \mathrm{an}^{-1}$ est respectée pour les tissus profonds. La limite annuelle pour la peau (prévention des effets non stochastiques) est, en effet, dix fois plus élevée : 0,5 Sv.

$\mathrm{Si}$, donc, l'irradiation externe était la seule nuisance radiologique on devrait avoir :

$$
H_{P} \leqq 0,05 \mathrm{~Sv} \cdot \mathrm{an}^{-1}
$$

en désignant par $H_{P}$ l'équivalent de dose maximal en profondeur dont une valeur correcte est donnée par la lecture du dosimètre individuel.

\subsubsection{Inhalation des poussières de minerai}

Bien qu'on ait trouvé dans quelques cas une certaine accumulation des trois descendants de fin de chaîne (plomb 210, bismuth 210 et polonium 210) engendrés par les produits de filiation à vie courte du radon 222 [3], on admet qu'en règle générale on retrouve dans les poussières de minerai tous les descendants de l'uranium 238 à l'équilibre séculaire avec leur père moins le radon 222 et ses quatre enfants à vie courte.

Le tableau III donne pour ces radionucléides les valeurs des limites annuelles d'incorporation et les limites dérivées de concentration dans l'air correspondantes. Ces valeurs sont tirées, sauf pour le plomb 210 et le bismuth 210 , de la Publication 30 de la C.I.P.R. [4]. Pour le plomb 210 et le bismuth 210 , la C.I.P.R. n'a pas encore publié de valeurs et celles qui ont été retenues ont été calculées par le National radiological protection board (N.R.P.B.) [5]. Les valeurs recommandées par la C.I.P.R. seront retenues dans les textes réglementaires en préparation.

L'application de la formule bien connue des mélanges :

$$
\frac{1}{(\mathrm{LAI}) \text { mélange }}=\sum_{i} \frac{\alpha_{i}}{(\mathrm{LAI})_{i}},
$$

où $\alpha_{i}$ est la proportion en activité du radionucléide $i$ dans le mélange et (LAI) ${ }_{i}$ la limite annuelle d'incorporation pour ce radionucléide, permet d'obtenir la limite annuelle d'incorporation pour les poussières de minerai, dont l'activité est supposée mesurée en $\alpha$ :

$$
1,5 \cdot 10^{3} \mathrm{~Bq}(\alpha) \quad\left({ }^{2}\right) .
$$

$\left({ }^{2}\right)$ Voir $\left({ }^{3}\right)$.

VOL. $16-\mathrm{N}^{\circ} 1$ 
Un calcul plus rigoureux tenant compte des équivalents de dose délivrés à chaque organe conduit à une valeur plus exacte :

$$
1,8 \cdot 10^{3} \mathrm{~Bq}(\alpha),
$$

ce qui donne en appliquant la formule de la C.I.P.R. 30 (fondée sur 2000 heures de travail par an et un débit respiratoire au travail de $1,2 \mathrm{~m}^{3}$ par heure) :

$$
\mathrm{LDCA}=\frac{\mathrm{LAI}}{2,4 \cdot 10^{3}}\left(\mathrm{~Bq} \cdot \mathrm{m}^{-3}\right),
$$

une limite dérivée de concentration dans l'air de :

$$
0,8 \mathrm{~Bq} \cdot \mathrm{m}^{-3} \text { soit } 22 \cdot 10^{-15} \mathrm{Ci}(\alpha) \cdot 1^{-1} \quad\left({ }^{3}\right) .
$$

Ce sont les limites individuelles qu'il faudrait respecter si l'inhalation des poussières de minerai était la seule nuisance radiologique.

\subsubsection{Inhalation des produits de filiation du radon 222}

La détermination d'une limite individuelle pour l'inhalation du radon 222 et de ses produits de filiation s'est heurtée, depuis 30 ans, à des difficultés considérables. Les raisons en sont multiples :

- la nature même des produits de filiation à vie courte du radon qui s'engendrent rapidement les uns les autres et passent selon des lois mal connues de l'état d'ions libres à l'état d'ions fixés sur des poussières;

- les mécanismes complexes de leur dépôt aux différents niveaux de l'appareil respiratoire;

- la dynamique compliquée de l'épuration pulmonaire;

- la présence du mucus dont les épaisseurs aux différents niveaux bronchiques sont mal connues;

- le fait qu'il n'y a aucune certitude sur l'importance respective des différents tissus de l'arbre respiratoire dans le phénomène d'induction des affections malignes du poumon par les rayonnements ionisants;

Toutes ces difficultés ont fait obstacle à l'établissement d'une relation simple entre exposition au radon et dose reçue au niveau des poumons.

Le problème revêtait pourtant un caractère particulièrement important car, de longue date, on a observé une augmentation significative des carcinomes pulmonaires parmi les mineurs d'uranium, principalement dans les mines de pechblende du Schneeberg et de Joachimsthal [6-9], dans les mines du Colorado aux États-Unis [10-19] ainsi que dans les mines de Tchécoslovaquie [20-23].

$\left({ }^{3}\right)$ Ces valeurs sont données à titre provisoire. Elles seront à revoir après parution des « Part 2 et Part 3 " de la publication 30 de la C.I.P.R. De plus, les pouvoirs publics auront à se prononcer sur la façon d'arrondir la valeur calculée. 
On pouvait penser que les études épidémiologiques menées sur ces populations de mineurs conduiraient rapidement à la connaissance de relation entre l'exposition et l'augmentation des cancers du poumon; en fait, ce résultat n'est pas encore parfaitement acquis en raison de nombreuses incertitudes concernant le rôle joué par le tabac, les gaz de combustion rejetés par les diesels, les métaux cancérigènes présents dans le minerai de certaines mines et surtout en raison des incertitudes sur les concentrations en radon et en produits de filiation auxquelles les mineurs étaient exposés à des époques où la surveillance de l'atmosphère des mines n'était pas aussi précise et stricte qu'elle l'est aujourd'hui.

Malgré toutes ces difficultés, la C.I.P.R., qui au cours de ces 15 dernières années, a créé plusieurs groupes de travail pour suivre l'évolution des connaissances sur ce sujet, a recommandé des limites dont l'expression a évolué au cours du temps en fonction des résultats acquis dans l'approfondissement de ces connaissances. Retraçons rapidement ces principales étapes.

\subsubsection{Les recommandations de 1955}

En 1955 [24] la C.I.P.R. recommandait une limite s'appliquant à la concentration moyenne (sur l'année) dans l'air « du radon en équilibre avec ses produits de filiation " pour une exposition correspondant aux heures de travail. Cette limite se dénommait concentration maximale admissible (CMA) pour une exposition professionnelle. Elle était fixée à :

$3.10^{-7}$ curie par mètre cube d'air $\left(\mathrm{Ci} . \mathrm{m}^{-3}\right)$ soit environ $10^{4}$ becquerels par mètre cube d'air $\left(\mathrm{Bq} \cdot \mathrm{m}^{-3}\right)$.

Aucune relation quantitative n'était donnée entre cette valeur de la CMA et l'équivalent de dose reçu par l'organe critique : le poumon. Cependant, le contexte de ces recommandations de 1955 permettait de penser que cette limite assurait, dans l'esprit de la commission, le respect d'une limite de dose hebdomadaire moyenne de $0,3 \mathrm{rem}\left(3 \cdot 10^{-3} \mathrm{~Sv}\right)$.

\subsubsection{Les recommandations de 1959}

Des travaux entrepris vers la même époque mirent nettement en évidence l'importance que jouaient les produits de filiation formés dans l'air par rapport au gaz radon lui-même dans l'irradiation des tissus pulmonaires : la dose due au radon est négligeable devant celle que délivrent les produits de filiation inhalés.

D'autres études menées après 1955 conduisirent certains chercheurs à penser que la partie la plus exposée de l'appareil respiratoire humain était l'épithélium bronchique et des physiciens, réalisant un montage représentant la trachée et les grosses bronches, mirent en évidence le rôle que pouvaient jouer les atomes libres, c'est-à-dire les produits de filiation du radon non fixés sur les noyaux de condensation ou des poussières [25].

Essentiellement formés d'atomes de polonium 218, ces atomes libres se déposaient dans les voies supérieures des poumons et étaient responsables de la plus grande part de la dose délivrée au niveau de l'épithélium bronchique.

VOL. $16-\mathrm{N}^{\circ} 1$ 
Dans ses recommandations de 1959 (publication 2) [26] la C.I.P.R. se fondant sur ces travaux, présentait une formule reliant la valeur de la concentration maximale admissible pour le radon 222 pour une exposition professionnelle de 40 heures de travail par semaine, à la fraction $f$ d'ions de polonium 218 non fixés à des noyaux de condensation dans l'air inhalé :

$$
\mathrm{CMA}=\frac{3 \cdot 10^{-6}}{1+1000 f} .
$$

On voit que la valeur numérique de la CMA peut ainsi varier de $3 \cdot 10^{-6}$ à $3 \cdot 10^{-9} \mathrm{Ci} \cdot \mathrm{m}^{-3}$ selon que $f$ varie de 0 à 1.

Sans insister sur les difficultés que présente la détermination physique de ce qu'on appelle ions libres et, par conséquent, sur les difficultés de la mesure, notons que les valeurs du paramètre $f$ trouvées dans la plupart des mines exploitées en Europe [27-28] ont conduit la Communauté Européenne à adopter la valeur qui figure dans ses directives :

$$
3 \cdot 10^{-7} \mathrm{Ci} \cdot \mathrm{m}^{-3} \text {. }
$$

Il s'agit de la limite concernant le radon en équilibre avec ses produits de filiation. Dans la mesure où il n'y a pas équilibre, il y a lieu d'appliquer à la concentration mesurée du radon un facteur correctif tenant compte de l'importance du déséquilibre. Cette valeur de la CMA doit être respectée en moyenne sur 3 mois.

\subsubsection{3. Évolution des connaissances entre 1959 et 1980}

Depuis 1959, cependant, les recherches se sont poursuivies tant dans l'élaboration de modèles permettant une meilleure évaluation des doses reçues par l'appareil respiratoire que dans les études épidémiologiques sur la mortalité par cancer du poumon chez les mineurs des mines du Colorado et de Tchécoslovaquie. Tout d'abord, le besoin se fit sentir de disposer d'une grandeur physique qui rende mieux compte de la nocivité des produits de filiation du radon, indépendamment du radon lui-même et de l'équilibre de ses produits de filiation. C'est ainsi que se généralisa l'usage d'une grandeur préconisée par le U.S. Public Health Service et qui, négligeant l'apport des rayonnements $\beta$, est appelée énergie potentielle $\alpha$ des produits de filiation du radon dans l'air [29]. Cette notion se définit comme la somme des énergies des particules $\alpha$ du polonium 218 et du polonium 214 qui auront été émises lorsque tous les produits de filiation à vie courte (quelle que soit leur proportion) contenus à un instant donné dans un certain volume d'air, choisi comme unité, se seront désintégrés. Il s'agit donc d'une énergie par unité de volume qui doit s'exprimer, dans le système international, en joules par mètre cube $\left(\mathrm{J} \cdot \mathrm{m}^{-3}\right)\left({ }^{4}\right)$.

$\left({ }^{4}\right)$ Une terminologie correcte exigerait que cette grandeur soit appelée " énergie potentielle $\alpha$ volumique $"$. 
L'unité adoptée aux U.S.A., et dont l'usage s'est répandu, est l'énergie potentielle $\alpha$ des produits de filiation en équilibre avec le radon dans $11 \mathrm{~d}$ 'air où l'activité volumique du radon est de $10^{-10} \mathrm{Ci} \cdot 1^{-1}\left(10^{-7} \mathrm{Ci} \cdot \mathrm{m}^{-3}\right)$ soit $1,3 \cdot 10^{5} \mathrm{MeV} \cdot 1^{-1}$ ou $2,08 \cdot 10^{-8} \mathrm{~J} \cdot 1^{-1}$. On l'appelle working level, niveau de travail (WL).

Pour évaluer l'exposition de l'homme en terme d'incorporation, il faut faire intervenir le débit respiratoire $B$ et le temps $t$ pendant lequel l'homme reste dans l'atmosphère dont l'énergie potentielle $\alpha$ a une valeur déterminée $W$. On obtient alors l'énergie incorporée en faisant le produit :

$$
\underset{\left(\mathrm{J} \cdot \mathrm{m}^{-3}\right)}{W} \cdot \underset{\left(\mathrm{m}^{3} \cdot \mathrm{h}^{-1}\right)}{B} \cdot t=I
$$

dont la dimension est bien celle d'une énergie.

Là encore, l'usage d'une grandeur pratique anglo-saxonne s'est répandu, qui fait abstraction du débit respiratoire (dont on admet qu'il est une constante) et se réduit au produit de l'énergie potentielle $\alpha$ dans l'air multipliée par le temps de présence, ce qui donne l'expression suivante, en unités anglo-saxonnes, de l'exposition $E$ :

$$
\underset{(\text { WL })}{W} \cdot \underset{(\text { Month }=170 \mathrm{~h})}{t}=\underset{(\mathrm{WLM})}{E},
$$

WLM = working level month (niveau de travail mois).

Dans cette expression, le mois correspond à 170 heures de travail, ce qui est une valeur moyenne du temps mensuel de travail moyenné sur l'année.

Avec le débit respiratoire adopté par la Publication C.I.P.R. 30 [4] $\left(1,2 \mathrm{~m}^{3} \cdot \mathrm{h}^{-1}\right.$ pendant les heures de travail $)$, les relations entre la terminologie anglo-saxonne et celle du système international de grandeurs et d'unités sont les suivantes :

1 WL correspond à : $2,08 \cdot 10^{-5} \mathrm{~J} \cdot \mathrm{m}^{-3}$ d'air;

1 WLM correspond à : $4,25 \cdot 10^{-3} \mathrm{~J}$ inhalé.

Toutes ces considérations peuvent paraîtrent bien austères et se rapporter à un domaine trop technique. Cela est peut-être vrai, mais il faut prendre conscience que ces notions sont fondamentales et indispensables pour qui veut comprendre les rapports qui existent entre les limites fixées pour le radon par la C.I.P.R. en 1959, celles adoptées par les U.S.A. qui, en 1974, au vu des résultats de leur enquête épidémiologique, décidèrent de réduire à 4 WLM par an l'exposition professionnelle au radon, et celles, enfin, que vient de recommander la C.I.P.R. dans un communiqué publié à l'issue de sa réunion de Brighton de mars 1980 .

Cela est également vrai, si on veut suivre les recherches poursuivies sur les modèles dosimétriques qui se sont efforcées d'évaluer l'équivalent de dose reçu par les différentes régions de l'arbre pulmonaire, ou l'arbre pulmonaire lui-même pris comme un tout, lorsque l'exposition est de 1 WLM. De plus, les études 
épidémiologiques sur la mortalité par cancer du poumon chez les mineurs, expriment toujours l'exposition au radon en WLM cumulés sur la vie professionnelle du mineur.

Il ne peut être question ici de retracer, même succinctement, le déroulement des très nombreux travaux publiés entre 1959 et 1980 tant sur l'expérimentation animale, où la France occupe une place de choix avec les recherches des équipes des Drs Lafuma et Chameaud [30-34], que sur les modèles dosimétriques et sur les données épidémiologiques. Soulignons simplement deux idées qui ont paru se dégager dans ces deux derniers domaines, malgré l'insuffisance des données :

a) La prise en considération, selon les principes de la C.I.P.R., de l'équivalent de dose reçu au niveau de l'ensemble de l'appareil respiratoire plutôt que de l'équivalent de dose reçu au niveau de certaines cellules dont on pouvait penser que leur exposition aux rayonnements était déterminante dans l'induction des cancers du poumon. Les valeurs de l'équivalent de dose au poumon par WLM varient quelque peu avec la proportion d'ions libres dans l'atmosphère, mais semblent se situer aux environs de $4 \cdot 10^{-2} \mathrm{~Sv}(4 \mathrm{rems})$, valeur calculée avec un facteur de qualité $Q=20$ pour les $\alpha$ [35].

b) L'idée que les résultats des deux enquêtes épidémiologiques (américaine et tchécoslovaque), bien que conduisant à des estimations quantitatives du risque assez différentes, ne sont cependant pas incompatibles si l'on tient compte des différences entre les populations étudiées. Bien qu'aucune augmentation significative du nombre de morts par cancer du poumon n'ait pu être mise en évidence au dessous de 120 WLM, leur interprétation peut conduire à une valeur du facteur de risque qui serait, au maximum, dans l'hypothèse d'une relation linéaire et sans seuil, de l'ordre de :

\section{$2.10^{-4}$ par WLM [36]}

soit :

$$
4 \cdot 10^{-2} \text { par joule inhalé. }
$$

Ceci conduirait pour le poumon à un facteur de risque apparent (par sievert) plus élevé que celui qui a été retenu par la C.I.P.R. (tableau II) :

$$
0,5 \cdot 10^{-2} \mathrm{~Sv}^{-1} \text { au lieu de } 0,2 \cdot 10^{-2} \mathrm{~Sv}^{-1}
$$

mais apparent seulement, car l'interprétation des données ne peut pas distinguer entre l'action du radon, celle de l'irradiation externe et celle des autres toxiques non radioactifs qui peuvent avoir une action synergétique comme le tabac, les fumées de diesel, les poussières de silice, de métaux, etc. Ainsi, l'augmentation de la fréquence des cancers pulmonaires est attribuée à la seule action du radon, ce qui est une interprétation très pessimiste.

Nous allons voir que la C.I.P.R. est bien consciente de ces insuffisances dans les conclusions des études épidémiologiques et qu'elle le mentionne explicitement dans le «communiqué » où elle recommande une nouvelle limite pour l'exposition professionnelle au radon et à ses produits de filiation [37].

3.4.3.4. Recommandations de mars 1980 sur l'exposition professionnelle au radon 222 et à ses produits de filiation 
« La Commission est parvenue à une conclusion en ce qui concerne la limite qui convient pour l'exposition professionnelle au radon et à ses produits de filiation.

" Pour la détermination de cette limite, la Commission a pris comme base le niveau de risque correspondant à la limite actuelle de $50 \mathrm{mSv}$ par an fixée pour l'équivalent de dose effectif. Il y a plusieurs façons d'établir une relation entre la quantitée inhalée de radon et de ses produits de filiation, et le niveau de risque. On a utilisé la méthode dosimétrique dont on s'est servi dans la Publication 30 pour la plupart des substances radioactives ainsi qu'une méthode similaire quelque peu différente pour tenir compte des problèmes particuliers que posent les produits de filiation à vie courte du radon. Les études épidémiologiques ont constitué une troisième méthode. Il y a un accord raisonnablement étroit entre les résultats de ces trois méthodes et la Commission recommande une limite qui se situe au niveau le plus faible des résultats dosimétriques et qui est en accord avec les conclusions des études épidémiologiques.

"Ces conclusions ne se rapportent pas spécifiquement au radon car elles concernent les conséquences de l'exposition à tout l'environnement minier qui contient des agents potentiellement nocifs non radioactifs. Un rapport de la Commission destiné à être publié est en cours de préparation.

« La limite annuelle d'incorporation par inhalation (LAI) recommandée pour les produits de filiation du radon 222 est, en terme d'énergie potentielle $\alpha$ inhalée : $0,02 \mathrm{~J}$ par an.

«La concentration dérivée dans l'air correspondante (voir Publication 30), exprimée en utilisant les unités pratiques largement en usage auparavant est alors : 0,4 niveau de travail (WL).

"Le système de limitation des doses adopté par la Commission exige que l'on fasse la somme de l'exposition à l'irradiation externe et des incorporations de substances radioactives. Dans le cas particulier de l'exposition dans les mines d'uranium, cette règle d'additivité revient à exiger que l'inhalation du radon et de ses produits de filiation soit maintenue à une valeur inférieure à la limite recommandée et, ce, dans une mesure qui dépend de l'exposition à l'irradiation externe et de l'exposition aux poussières de minerai. Une réduction de $20 \mathrm{p}$. cent est courante.

"Ces recommandations sont destinées aux autorités compétentes pour une application générale et leur application dans certains cas particuliers peut ne pas être pertinente. La Commission est consciente du fait que les conditions de certaines mines sont telles qu'il peut être impossible d'opérer dans les limites combinées recommandées par la Commission en les appliquant sur une base strictement annuelle. Les autorités nationales devront alors décider de la meilleure façon de régler ces situations peu courantes, mais difficiles ".

Il faut noter dans ce texte le rappel explicite de l'exigence du cumul des risques et l'idée développée dans le dernier alinéa que ces limites peuvent, dans certains cas, être difficiles à appliquer et qu'une réglementation nationale prévoyant une application sur une base qui ne serait pas strictement annuelle, ne serait pas en opposition avec les recommandations de la Commission.

$\mathrm{Si}$ les produits de filiation du radon constituaient la seule nuisance radiologique (associée cependant aux autres nuisances non radiologiques) des mines, la limite à respecter serait donc une limite d'incorporation annuelle par inhalation de $0,02 \mathrm{~J}$, ce qui correspond à une limite dérivée de concentration dans l'air de $0,4 \mathrm{WL}\left(8,3 \cdot 10^{-6} \mathrm{~J} . \mathrm{m}^{-3}\right)$ pour 2000 heures d'exposition par an, le débit respiratoire étant de $1,2 \mathrm{~m}^{3} \cdot \mathrm{h}^{-1}$. Si nécessaire, les pouvoirs publics pourront décider de mesures adaptées à certains cas particuliers.

Il y a lieu de remarquer que la Commission n'a formulé de recommandations que pour l'exposition professionnelle au radon et à ses produits de filiation. Étant donné que la limite qu'elle recommande est en accord avec les conclusions des études épidémiologiques dont il est bien précisé qu'elles rendent compte du risque pour l'environnement minier, donc tiennent compte 
des synergies avec tous les toxiques propres aux mines, on peut se demander s'il serait bien justifié d'appliquer aux personnes du public une limite 10 fois plus faible, comme c'est l'usage pour les autres radionucléides. A première vue une telle limite serait d'une sévérité difficile à justifier.

\subsection{Le CUMUl DES RISQUES}

Rappelons enfin, comment devrait s'appliquer aux trois risques radiologiques des mines la formule du cumul des risques.

$\mathrm{Si}$ on adopte les conventions symboliques suivantes :

$H_{p}=$ équivalent de dose annuel maximal en profondeur due à l'irradiation externe $(\mathrm{Sv})$;

$I_{P M}=$ activité $\alpha$ de poussière de minerai inhalée dans l'année $(\mathrm{Bq})$;

$J_{R}=$ énergie potentielle $\alpha$ des produits de filiation du radon 222 inhalée dans l'année $(\mathrm{J})$,

on devrait avoir, en admettant que la limite dérivée de concentration dans l'air précédemment calculée pour les poussières de minerai, soit retenue :

$$
\frac{H_{p}}{0,05}+\frac{I_{P M}}{1,8 \cdot 10^{3}}+\frac{J_{R}}{0,02} \leqq 1 .
$$

\section{BIBLIOGRAPHIE}

[1] Recommandations de la Commission internationale de protection radiologique, Publication C.I.P.R. 26 (1977) compte tenu des modifications adoptées à Stockholm en mai 1978. Oxford, Pergamon Press, 1980.

[2] Pochin E. E. Why be quantitative about radiation risk estimates? Washington, N.C.R.P., 1978.

[3] PRADEL J. Les contrôles atmosphériques dans les mines d'uranium. In: Symposium sur la mesure des doses d'irradiation, Stockholm, 1967, Paris, O.C.D.E., 1967.

[4] I.C.R.P. Limits for intakes of radionuclides by workers, I.C.P.R. Publication 30 part 1, Oxford, Pergamon press, 1979.

[5] Adams N., Hunt B. W., Reissland J. A. Annual limits of intake of radionuclides for workers, NRPB-R82, 1978.

[6] Pirchan A., SiKL H. Cancer of the lung in miners of Jachymov (Joachimsthal): report of cases observed in 1929-1930. Amer. J. Cancer, 1932, 16, 681-722.

[7] LORENZ E. Radioactivity and lung cancer: a critical review of lung cancer in the miners of Schneeberg and Joachimstal. J. Natl. Cancer Inst., 1944, 5, 1-15.

[8] Rostoskı O., Saupe E., Schmorl G. Die Bergkrankheit der Erzbergleute in Schneeberg in Sachsen (Scheenberger Lungenkrebs). Z. Krebsforsch., 1926, 23, 360-384.

[9] SIKL H. The present status of knowledge about Jachymov disease (Cancer of the lung in the miners of the radium mines). Unio Intern. Cont. Cancr. Acta, 1950, 6, 1366-1375.

[10] Archer V. E., Magnuson H. J., Holaday D. A. et al. Hazards to health in uranium mining and milling, 46th Annual meeting of the Industrial medical association, Los Angeles, April 13, 1961. J. Occup. Med., 1962, 4, 55-60. 
[11] Governor's Conference. Health hazards in uranium mines, P.H.S. Publication, $\mathrm{n}^{\circ} 843$. Washington, Public health service, 1961.

[12] Wagoner J. K., Archer V. E., CARroll D. E. et al. Cancer mortality patterns among US uranium miners and millers, 1950 through 1962. J. Natl. Cancer Inst., 1964, 32, 787-801.

[13] WAGoner J. K., ArCher V. E., Lundin F. E. et al. Radiation as the cause of lung cancer among uranium miners. New Engl. J. Med., 1965, 273, 181-188.

[14] Saccomanno G., Archer V. E., Saunders R. P. et al. Lung cancer of uranium miners on the Colorado plateau. Health Phys., 1964, 10, 1195-1201.

[15] Lundin F. E., ARCher V. E., SMith E. M. et al. Lung cancer among U.S. uranium miners: current assessment of risk, presented to the American public health association, Miami, October 24, 1967.

[16] Lundin F. E., Lloyd J. W., SMith E. M. et al. Mortality of uranium miners in relation to radiation exposure, hard rock mining and cigarette smoking, 1950 through September 1967. Health Phys., 1969, 16, 571-578.

[17] LUNDIN F. E., WAGONER J. K., ARCHER V. E. Radon daughter exposure and respiratory cancer: quantitative and temporal aspects, NIOSH and NIEHS joint Monograph, $\mathrm{n}^{\circ} 1$. Springfield, National technical information service, 1971.

[18] ArCher V. E., Wagoner J. K., LUNDIN F. E. Lung cancers among uranium miners in the United States. Health Phys., 1973, 25, 351-373.

[19] Archer N. E., Wagoner J. K., Hyg S. D., Lundin F. E. Uranium mining and cigarette smoking effect on man. J. Occup. Med., 1973, 15, 204-211.

[20] SEvC J., PLACEK V. Radiation induced lung cancer: relation between lung cancer and long term exposure to radon daughters. In: Proc. 6th Conf. on rad. hyg., C.S.S.R., p. 305, 1973.

[21] Sevc J., Kunz E., PLACEK V. Lung cancer in uranium miners and long term exposure to radon daughters products. Health Phys., 1976, 30, 433-437.

[22] Kunz E., Sevc J., PlaceK V. Lung cancer in uranium miners (methodological aspects). Health Phys. 1978, 35, 579-580.

[23] Kunz E., Sevc J., Placek V., Horacek J. Lung cancer in man in relation to different time distribution of radiation exposure. Health Phys., 1979, 39, 699-706.

[24] I.C.R.P. Recommendations of the International commission on radiogical Protection, Br. J. Radiol., 1955, suppl. 6. Edition française : J. Radiol., 1956, 36, $\mathrm{n}^{\circ} 10$ bis.

[25] Chamberlain A. C., Dyson E. D. The dose to the trachea and bronchi from the decay products of radon and thoron. Br. J. Radiol. 1956, 29, 317-325.

[26] I.C.R.P.Report of Committee II on permissible dose for internal radiation. London, Pergamon press, 1959. Edition française : Paris, Gauthier-Villars, 1966.

[27] Billard F., Miribel J., Madel.aine G., Pradel J. In: Radiological health and safety in mining and milling of nuclear materials. Vienne, A.I.E.A., 1964, vol. 1, 411-424.

[28] Chapuis A., Lopez A., Fontan J., Madelaine G. Détermination de la fraction d'activité existant sous forme de RaA non attaché dans l'atmosphère d'une mine d'uranium. Health Phys. $1973,25,59-65$.

[29] États-Unis. Public health service. Control of radon and daughters in uranium mines and calculations on biologic effets U.S.P.H.S. Publication, $\mathrm{n}^{\circ} 494,1957$.

[30] Chameaud J., Perraud R., Lafuma J., Masse R. Risques et nuisances des mines d'uranium, prévention médicale. In : O.I.T., C.E.A., O.M.S. et A.I.E.A. Radiation protection in mining and milling of uranium and thorium, Bordeaux, 9-11 septembre 1974. Geneva, International Labour Office, 1976, 277-285.

vOL. $16-\mathrm{N}^{\circ} 1$ 
[31] Lafuma J., Chameaud J., Perraud R., Masse R., Nenot J. C., Morin M. Étude expérimentale de la comparaison de l'action toxique sur les poumons du radon 222 et de ses produits de filiation avec les émetteurs $\alpha$ de la série des actinides. In : O.I.T., C.E.A., O.M.S. et A.I.E.A. Radiation protection in mining and milling of uranium and thorium, Bordeaux, 911 septembre 1974. Geneva, International Labour Office, 1976, 43-53.

[32] Lafuma J. Cancers pulmonaires induits par différents émetteurs $\alpha$ inhalés. In: Late biological effects of ionizing radiation, Vienne, A.I.E.A., 1978, vol. 2, 531-541.

[33] Chameaud J., Perraud R., Chretien J., Masse M., Lafuma J. Étude expérimentale de l'action combinée de la fumée de cigarettes et du dépôt actif du radon 222. In: Late biological effects of ionizing radiation, Vienne, A.I.E.A., 1978, vol 2, 429-436.

[34] Chameaud J., Perraud R., Masse R., Lafuma J. Cancers induced by radon 222 in the rat. Specialist meeting on the assessment of radon and daughter exposure and related biological effects, Rome, 3-8 March 1980.

[35] Mechali D. Inhalation du radon et de ses produits de filiation : doses et effets pathologiques In: Radiation protection, 5th congress I.R.P.A., Jérusalem, 9-14 mars 1980, vol. 2, 957-964.

[36] JACOBI W. Interpretation of measurements in uranium mines: dose evaluation and biomedical aspects. In: La dosimétrie individuelle et la surveillance de l'atmosphère en ce qui concerne le radon et ses produits de filiation, Elliot Lake, 4-8 octobre 1976, Paris, O.C.D.E., 1977, 33-48.

[37] I.C.R.P. Statement issued after Brighton 1980 meeting. Ann. I.C.R.P., 1980, 24, n³/4. Health Phys., 1980, 39, 377-387. En français : Radioprotection, 1980, 15, 239-245. 